\title{
Quantum Brain: A Recurrent Quantum Neural Network Model to Describe Eye Tracking of Moving Targets
}

\author{
Laxmidhar Behera, ${ }^{1}$ Indrani Kar, ${ }^{1}$ and Avshalom Elitzur ${ }^{2}$ \\ ${ }^{1}$ Department of Electrical Engineering, Indian Institute of Technology, Kanpur 208016 , UP, INDIA \\ ${ }^{2}$ Unit of Interdisciplinary Studies, Bar-IIan University, 52900 Ramat-Gan, Israel
}

\begin{abstract}
A theoretical quantum brain model is proposed using a nonlinear Schroedinger wave equation. The model proposes that there exists a quantum process that mediates the collective response of a neural lattice (classical brain). The model is used to explain eye movements when tracking moving targets. Using a Recurrent Quantum Neural Network(RQNN) while simulating the quantum brain model, two very interesting phenomena are observed. First, as eye sensor data is processed in a classical brain, a wave packet is triggered in the quantum brain. This wave packet moves like a particle. Second, when the eye tracks a fixed target, this wave packet moves not in a continuous but rather in a discrete mode. This result reminds one of the saccadic movements of the eye consisting of 'jumps' and 'rests'. However, such a saccadic movement is intertwined with smooth pursuit movements when the eye has to track a dynamic trajectory. In a sense, this is the first theoretical model explaining the experimental observation reported concerning eye movements in a static scene situation. The resulting prediction is found to be very precise and efficient in comparison to classical objective modeling schemes such as the Kalman filter.
\end{abstract}

PACS numbers:

\section{INTRODUCTION}

Information processing in the brain is mediated by the dynamics of large, highly interconnected neuronal populations. The activity patterns exhibited by the brain are extremely rich; they include stochastic weakly correlated local firing, synchronized oscillations and bursts, as well as propagating waves of activity. Perception, emotion etc. are supposed to be emergent properties of such a complex nonlinear neural circuit.

Instead of considering one of the conventional neural architectures [1, 2, 3, 4, 5], an alternative neural architecture is proposed here for neural computing. Indeed, there are certain aspects of brain functions that still appear to have no satisfactory explanation. As an alternative, researchers [6, 7, 8, 9] are investigating whether the brain can demonstrate quantum mechanical behavior. According to current research, microtubules, the basic components of neural cytoskeleton, are very likely to possess quantum mechanical properties due to their size and structure. The tubulin protein, which is the structural block of microtubules, has the ability to flip from one conformation to another as a result of a shift in the electron density localization from one resonace orbital to another. These two conformations act as two basis states of the system according to whether the electrons inside the tubuline hydrophobic pocket are localized closer to $\alpha$ or $\beta$ tubulin. Moreover the system can lie in a superposition of these two basis states, that is, being in both states simultaneously, which can give a plausible mechanism for creating a coherent state in the brain. To give credence to the possibility of existence of a quantum brain, Penrose ([10]) argued that the human brain must utilize quantum mechanical effects when demonstrating problem solving feats that cannot be explained algorithmically.

In this paper, instead of going into biological details of the brain, we propose a theoretical quantum brain model. The model is referred to as Recurrent Quantum Neural Network (RQNN). An earlier version 11] of this model used a linear neural circuit to set up the potential field in which the quantum brain is dynamically excited. The present model uses a nonlinear neural circuit. This fundamental change in the architecture has yielded two novel features. The wave packets, $f(x, t)=|\psi(x, t)|^{2}$, are moving like particles. Here $\psi(x, t)$ is the solution of the nonlinear Schroedinger wave equation that describes the quantum brain model proposed in this paper to explain eye movements for tracking moving targets. The other very interesting observation is that the movements of the wave packets while tracking a fixed target are not continuous but discrete. These observations accord with the well-known saccadic movement of the eye [12]. In a way, our model is the first of its kind to explain the nature of eye movements in static scenes that consists of "jumps" (saccades) and "rests" (fixations). We expect this result to inspire other researchers to further investigate the possible quantum dynamics of the brain.

\section{A THEORETICAL QUANTUM BRAIN MODEL}

An impetus to hypothesize a quantum brain model comes from the brain's necessity to unify the neuronal response into a single percept. Anatomical, neurophysiological and neuropsychological evidence, as well as brain imaging using fMRI and PET scans, show that separate functional maps exist in the biological brain to code separate features such as direction of motion, location, color and orientation. How does the brain compute all this data to have a coherent perception? In this paper, a very simple model of a quantum brain is proposed where a col- 
lective response of a neuronal lattice is modeled using a Schroedinger wave equation as shown in FIG. 1 In this figure, it is shown that an external stimulus reaches each neuron in a lattice with a probability amplitude function $\psi_{i}$. Such a hypothesis would suggest that the carrier of the stimulus performs quantum computation. The collective response of all the neurons is given by the superposition equation:

$$
\psi=c_{1} \psi_{1}+c_{2} \psi_{2}+. .+c_{N} \psi_{N}=\sum_{i=0}^{N} c_{i} \psi_{i}
$$

We suggest that the time evolution of the collective response $\psi$ is described by the Schroedinger wave equation:

$$
i \hbar \frac{\partial \psi(x, t)}{\partial t}=-\frac{\hbar^{2}}{2 m} \nabla^{2} \psi(x, t)+V(x) \psi(x, t)
$$

where $2 \pi \hbar$ is Planck's constant, $\psi(x, t)$ is the wave function (probability amplitude) associated with the quantum object at space-time point $(x, t)$, and $m$ the mass of the quantum object. Further symbols such as $i$ and $\nabla$ carry their usual meaning in the context of the Schroedinger wave equation. Another way to look at our proposed quantum brain is as follows. A neuronal lattice sets up a spatial potential field $V(x)$. A quantum process described by a quantum state $\psi$ which mediates the collective response of a neuronal lattice evolves in the spatial potential field $V(x)$ according to equation (2). Thus the classical brain sets up a spatio-temporal potential field and the quantum brain is excited by this potential field to provide a collective response. In the next section, we present a possible eye-movement model for tracking moving target.

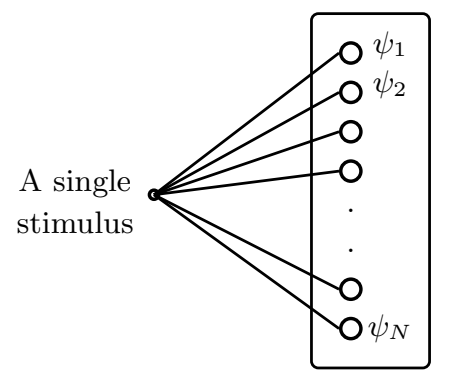

Collective Response

Neural Lattice $\psi=c_{1} \psi_{1}+c_{2} \psi_{2}+. .+c_{N} \psi_{N}$

FIG. 1: Quantum Brain - A Theoretical Model

\section{AN EYE TRACKING MODEL}

Let us consider a plausible biological mechanism for eye tracking using the quantum brain model proposed in section The mechanism of eye movements tracking a moving target consists of three stages as shown in FIG. 2 (i) stochastic filtering of noisy data that impact the eye sensors; (ii) a predictor that predicts the next spatial position of the moving target; and (iii) a biological motor control system that aligns the eye pupil along the moving targets trajectory. The biological eye sensor fans out the input signal $y$ to a specific neural lattice in the visual cortex. For clarity, Figure 2 shows a one-dimensional array of neurons whose receptive fields are excited by the signal input $y$ reaching each neuron through a synaptic connection described by a nonlinear map. The neural lattice responds to the stimulus by setting up a spatial potential field, $V(x, t)$, which is a function of external stimulus $y$ and estimated trajectory $\hat{y}$ of the moving target:

$$
V(x, t)=\sum_{i=1}^{n} W_{i}(x, t) \phi_{i}(\nu(t))
$$

where $\phi_{i}($.$) is a Gaussian Kernel function, n$ represents the number of such Gaussian functions describing the nonlinear map that represents the synaptic connections, $\nu(t)$ represents the difference between $y$ and $\hat{y}$ and $W$ represents the synaptic weights as shown in FIG. 22 The Gaussian kernel function is taken as:

$$
\phi_{i}(\nu(t))=\exp \left(-\left(\nu(t)-g_{i}\right)^{2}\right)
$$

where $g_{i}$ is the center of the $i^{\text {th }}$ Gaussian function, $\phi_{i}$. This center is chosen from input space described by the input signal, $\nu(t)$, through uniform random sampling.

Our quantum brain model proposes that a quantum process mediates the collective response of this neuronal lattice which sets up a spatial potential field $V(x, t)$. This happens when the quantum state associated with this quantum process evolves in this potential field. The spatio-temporal evolution follows as per equation (2). We hypothesize that this collective response is described by a wave packet, $f(x, t)=|\psi(x, t)|^{2}$, where the term $\psi(x, t)$ represents a quantum state. In a generic sense, we assume that a classical stimulus in a brain triggers a wave packet in the counterpart 'quantum brain'. This subjective response, $f(x, t)$, is quantified using the following estimate equation:

$$
\hat{y}(t)=\int x(t) f(x, t) d x
$$

The estimate equation is motivated by the fact that the wave packet, $f(x, t)=|\psi(x, t)|^{2}$ is interpreted as the probability density function. Based on this estimate, $\hat{y}$, the predictor estimates the next spatial position of the moving target. To simplify our analysis, the predictor is made silent. Thus its output is the same as that of $\hat{y}$. The biological motor control is commanded to fixate the eye pupil to align with the target position, which is 
predicted to be at $\hat{y}$. Obviously, we have assumed that biological motor control is ideal.

After the above mentioned simplification, the closed form dynamics of the model described by Figure 2 becomes:

$$
\begin{aligned}
i \hbar \frac{\partial \psi(x, t)}{\partial t}= & -\frac{\hbar^{2}}{2 m} \nabla^{2} \psi(x, t)+ \\
& \zeta G\left(y(t)-\int x|\psi(x, t)|^{2} d x\right) \psi(x, t)
\end{aligned}
$$

where $G($.$) is a Gaussian kernel map introduced to non-$ linearly modulate the spatial potential field that excites the dynamics of the quantum object. In fact $\zeta G()=$. $V(x, t)$ where $V(x, t)$ is given in equation (3).

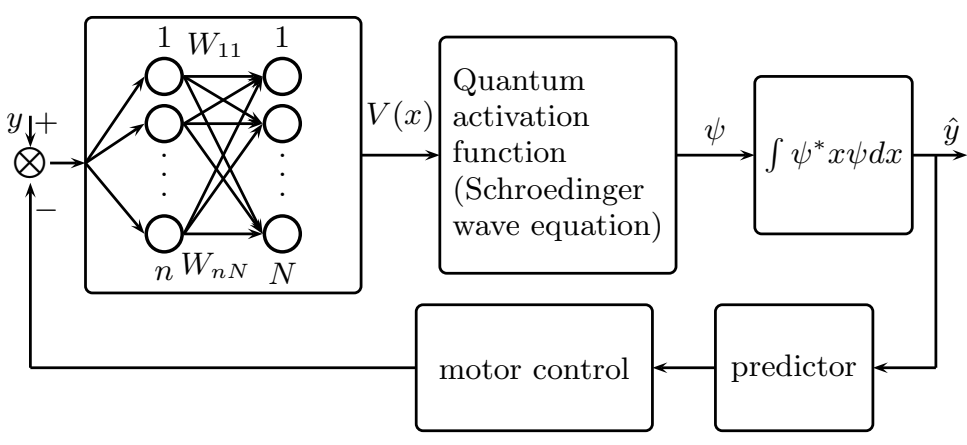

FIG. 2: Conceptual framework for the Recurrent Quantum Neural Networks

The nonlinear Schroedinger wave equation given by equation (6) is one-dimensional with cubic nonlinearity. Interestingly, the closed form dynamics of the Recurrent Quantum Neural Network (equation (6) ) closely resembles a nonlinear Schroedinger wave equation with cubic nonlinearity studied in quantum electrodynamics [13]:

$$
\begin{aligned}
i \hbar \frac{\partial \psi(x, t)}{\partial t}= & \left(-\frac{\hbar^{2}}{2 m} \nabla^{2}-\frac{e^{2}}{r}\right) \psi(x, t)+ \\
& e^{2} \int \frac{\psi(x, t)\left|\psi\left(x^{\prime}, t\right)\right|^{2}}{\left|x-x^{\prime}\right|} d x^{\prime}
\end{aligned}
$$

where $m$ is the electron mass, $e$ the elementary charge and $r$ the magnitude of $|x|$. Also, nonlinear Schroedinger wave equations with cubic nonlinearity of the form $\frac{\partial}{\partial t} \mathcal{A}(t)=c_{1} \mathcal{A}+c_{3}|\mathcal{A}|^{2} \mathcal{A}$, where $c_{1}$ and $c_{3}$ are constants, frequently appear in nonlinear optics [14] and in the study of solitons 15, 16, 17, 18].

In equation (6), the unknown parameters are weights $W_{i}(x, t)$ associated with the Gaussian kernel, mass $m$, and $\zeta$, the scaling factor to actuate the spatial potential field. The weights are updated using the Hebbian learning algorithm

$$
\frac{\partial W_{i}(x, t)}{\partial t}=\beta \phi_{i}(\nu(t)) f(x, t)
$$

where $\nu(t)=y(t)-\hat{y}(t)$.

The idea behind the proposed quantum computing model is as follows. As an individual observes a moving target, the uncertian spatial position of the moving target triggers a wave packet within the quantum brain. The quantum brain is so hypothesized that this wave packet turns out to be a collective response of a classical neural lattice. As we combine equations (6) and (8), it is desired that there exist some parameters $m, \zeta$ and $\beta$ such that each specific spatial position $x(t)$ triggers a unique wave packet, $f(x, t)=|\psi(x, t)|^{2}$, in the quantum brain. This brings us to the question whether the closed form dynamics can exhibit soliton properties. As pointed out above, our equation has a form that is known to possess soliton properties for a certain range of parameters and we just have to find those parameters for each specific problem.

We would like to reiterate the importance of the soliton properties. According to our model, eye tracking means tracking of a wave packet in the domain of the quantum brain. The biological motor control aligns the eye pupil along the spatial position of the external target that the eye tracks. As the eye sensor receives data $y$ from this position, the resulting error stimulates the quantum brain. In a noisy background, if the tracking is accurate, then this error correcting signal $\nu(t)$ has little effect on the movement of the wave packet. Precisely, it is the actual signal content in the input $y(t)$ that moves the wave packet along the desired direction which, in effect, achieves the goal of the stochastic filtering part of the eye movement for tracking purposes.

\section{SIMULATION RESULTS}

In this section we present simulation results to test target tracking through eye movement where targets are either fixed or moving.

For fixed target tracking, we have simulated a stochastic filtering problem of a dc signal embedded in Gaussian noise. As the eye tracks a fixed target, the corresponding dc signal is taken as $y_{a}(t)=2.0$, embedded in Gaussian noise with SNR (signal to noise ratio) values of $20 \mathrm{~dB}, 6$ $\mathrm{dB}$ and $0 \mathrm{~dB}$.

We compare the results with the performance of a Kalman filter [19] designed for this purpose. It should be noted that the Kalman filter has the a priori knowledge that the embedded signal is a dc signal whereas the RQNN is not provided with this knowledge. The Kalman filter also makes use of the fact that the noise is Gaussian and estimates the variance of the noise based on this assumption. Thus it is expected that the performance of the Kalman filter will degrade as the noise becomes nonGaussian. In contrast, the RQNN model does not make any assumption about the noise.

It is observed that there are certain values of $\beta, m$, $\zeta$ and $N$ for which the model performs optimally. A univariate marginal distribution algorithm [11] was used 

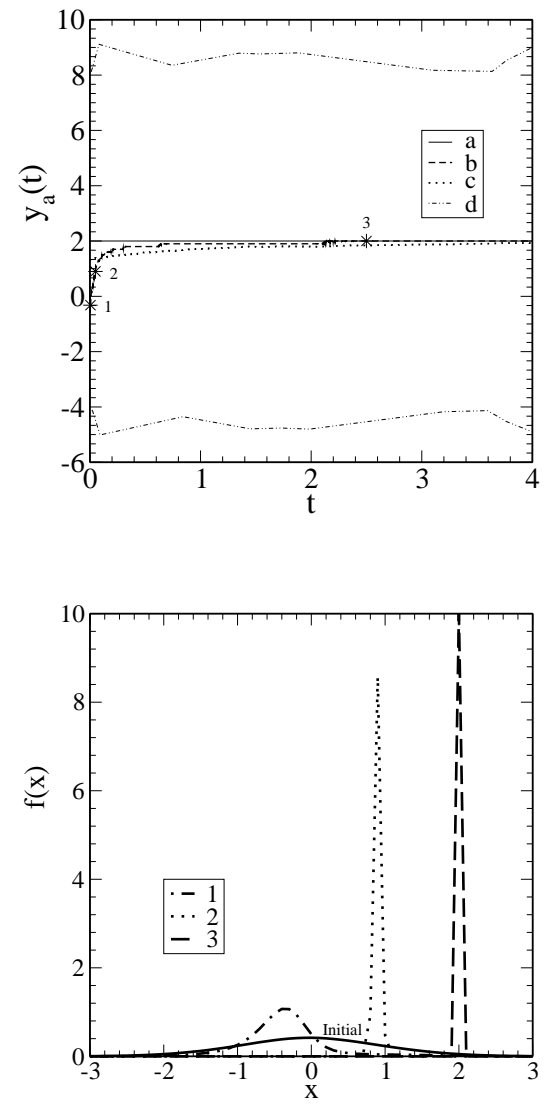

FIG. 3: (top) Eye tracking of a fixed target in a noisy environment of $0 \mathrm{~dB}$ SNR: 'a' respresents fixed target, 'b' represents target tracking using RQNN model and 'c' represents target tracking using a Kalman filter. The noise envelope is represented by the curve 'd'; (bottom) The snapshots of the wave packets at different instances corresponding to the marker points $(1,2,3)$ as shown in the top figure. The solid line represent the initial wave packet assigned to the Schroedinger wave equation.

to get near optimal parameters while fixing $N=400$ and $\hbar=1.0$. The selected values of these parameters are as follows for all levels of SNR:

$$
\beta=0.86 ; \quad m=2.5 ; \quad \zeta=2000 ;
$$

The comparative performance of eye tracking in terms of rms error for all the noise levels is shown in Table I. It is easily seen from Table I that the rms tracking error of RQNN is much less than that of the Kalman filter. Moreover, RQNN performs equally well for all the three categories of noise levels, whereas the performance of the Kalman filter degrades with the increase in noise level. In this sense we can say that our model performs the tracking with a greater efficiency compared to the Kalman filter. The exact nature of trajectory tracking is shown for $0 \mathrm{~dB}$ SNR in FIG. 3. In this figure, the noise enve-

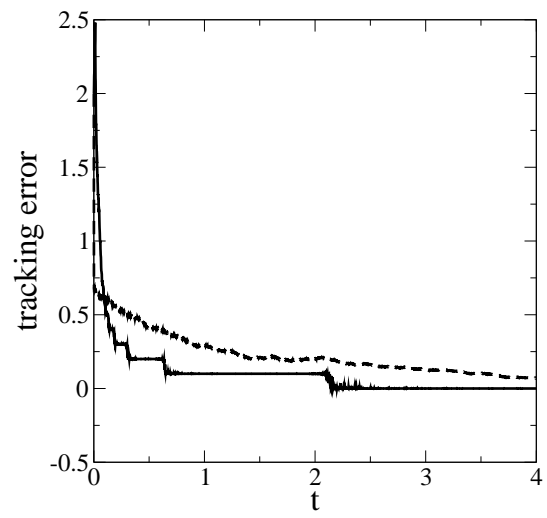

FIG. 4: The continuous line represents tracking error using RQNN model while the broken line represents tracking error using Kalman filter

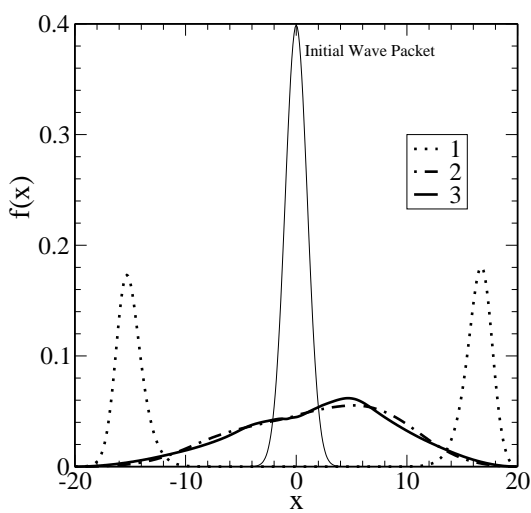

FIG. 5: Wave packet movements for RQNN with linear weights

lope is shown, and obviously its size is large due to a high noise content in the signal. The figure shows the trajectory of the eye movement as the eye focuses on a fixed target. To better appreciate the tracking performance, an error plot is shown in FIG. 4. Although Kalman filter tracking is continuous, the RQNN model tracking con-

TABLE I: Performance comparison between Kalman filter and RQNN for various levels of Gaussian noise

\begin{tabular}{lll}
\hline $\begin{array}{l}\text { Noise level RMS error } \\
\text { in dB }\end{array}$ & $\begin{array}{l}\text { RMS error } \\
\text { for Kalman filter }\end{array}$ & \begin{tabular}{l} 
for RQNN \\
\hline 20
\end{tabular} \\
\hline 0.0018 & 0.000040 \\
6 & 0.0270 & 0.000062 \\
0 & 0.0880 & 0.000090 \\
\hline
\end{tabular}


sists of 'jumps' and 'fixations'. As the alignment of the eye pupil becomes closer to the target position, the 'fixation' time also increases. Similar tracking behaviour was also observed for the SNR values of 20 and $6 \mathrm{~dB}$. These theoretical results are very interesting when compared to experimental results in the field of eye-tracking. In eyetracking experiments, it is known that eye movements in static scenes are not performed continuously, but consist of "jumps" (saccades) and "rests" (fixations). Eyetracking results are represented as lists of fixation data. Furthermore, if the information is simple or familiar, eye movement is comparatively smooth. If it is tricky or new, the eye might pause or even flip back and forth between images. Similar results are given by our simulations. Our model tracks the dc signal which can be thought of as equivalent to a static scene, in discrete steps rather than in a continuous fashion. This is very clearly understood from the tracking error in FIG. 4]

The other interesting aspect of the results is the movement of wave packets. In Figure 3 (bottom), snapshots of wave packets are plotted at different instances corresponding to marker points as shown along the desired trajectory. It can be noticed that a very flat initial Gaussian wave packet first moves to the left, and then proceeds toward the right until the mean of the wave packet exactly matches the actual spatial position. A similar pattern of movement of wave packets was also noticed in the case of 20 and $6 \mathrm{~dB}$ SNR. The wave packet movement is compared with our previous work [1] in FIG. [5 The initial wave packet in the previous model first splits into two parts, then moves in a continuous fashion, ultimately going into a state with a mean of approximately 2 but with high variance. In contrast, in the present model there is no splitting of the wave packet, movement is discrete and variance is also much smaller. Thus the soliton behavior of the present model is very much pronounced.

To analyze the eye movement following a moving target, a sinusoidal signal $y_{a}(t)=2 \sin 2 \pi 10 t$ is taken as the desired dynamic trajectory. This signal is embedded in $20 \mathrm{~dB}$ Gaussian noise. The parameter values for tracking this signal were fixed at $\beta=0.01, m=1.75$ and $\zeta=-250$. It is observed that during the training phase, the wave packet jumps from time to time, thus changing the tracking error until a steady state trajectory following is achieved. This feature is clearly understood from the tracking error plot which is shown in FIG. [6 In this figure, it is shown that the wave packet has jumped six times before the first smooth movement started. Again, this jump took place four times before the second smooth movement started and ultimately achieved a steady state. When the steady state is achieved, the tracking is efficient and the wave packet movement is continuous, as shown in FIG. 7 The snapshots of the wave packets are plotted for three different instances of time indicated by the marker points $(1,2,3)$ as shown in the trajectory tracking. When the signal is at position 1 , the corresponding wave packet has a mean at 0 . When the signal is at position 2 , the corresponding wave packet has a mean at +2 , and the mean of the wave packet moves to -2 when the signal goes to position 3 . This type of continuous movement of wave packet takes place after a series of 'jumps' of random nature. During continuous movement of the wave packets, trajectory tracking is smooth, denoted as smooth pursuit movement in the context of biological eye tracking. This theoretical result is very similar in nature as what has been observed experimentally [20].

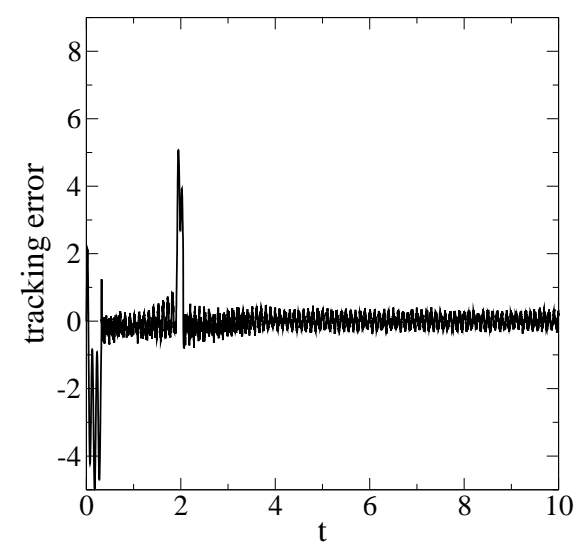

FIG. 6: Saccadic and pursuit movement of eye during dynamic trajectory following

\section{CONCLUSION}

The nature of eye movement has been studied in this article using the proposed RQNN model, where the predictor and motor control are assumed to be ideal. The most important finding is that our theoretical model of eye-tracking agrees with previously observed experimental results. The model predicts that eye movements will be of saccadic type while following a static trajectory. In the case of dynamic trajectory following, eye movement consists of saccades and smooth pursuits. In this sense, the proposed quantum brain concept in this paper is very successful in explaining the nature of eye movements. Earlier explanation [12] for saccadic movement has been primarily attributed to motor control mechanism whereas the present model emphasizes that such eye movements are due to decision making process of the brain - albeit quantum brain. Thus the significant contribution of this paper to explain biological eye-movement as a neural information processing event may inspire researchers to study quantum brain models from the biological perspective.

The other significant contribution of this paper is the prediction efficiency of the proposed model over the prevailing model. The stochastic filtering of a dc signal using RQNN is 1000 times more accurate compared to a Kalman filter. 

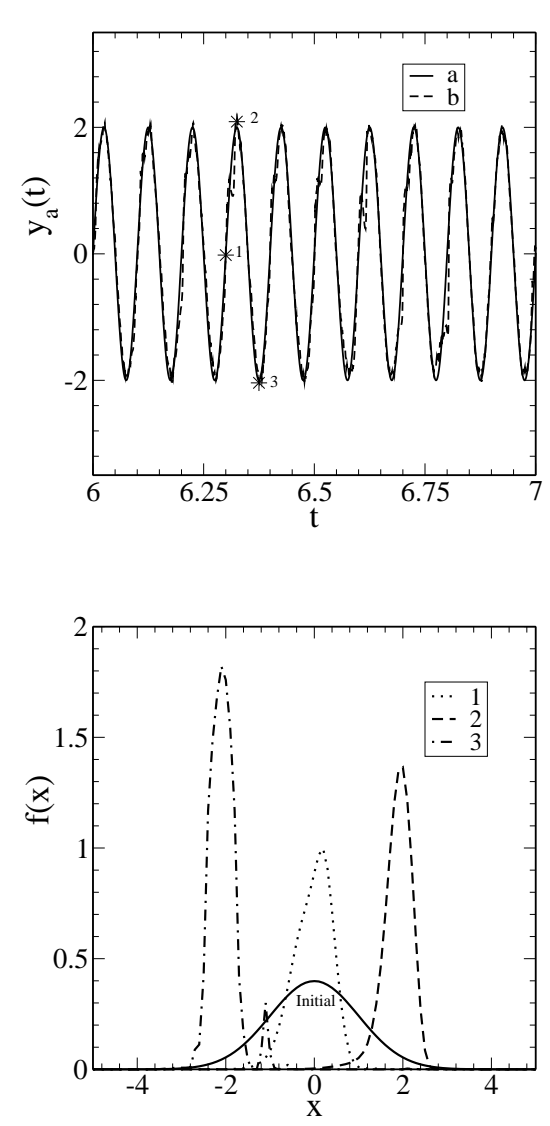

FIG. 7: (top) Eye tracking of a moving target in a noisy environment of 20dB SNR: 'a' respresents a moving target, 'b' represents target tracking using RQNN model; (bottom) The snapshots of the wave packets at different instances corresponding to the marker points $(1,2,3)$ as shown in the top figure. The solid line represents the initial wave packet assigned to the Schroedinger wave equation.
At this point the paper is silent about exact biological connection between classical and quantum brain since it is not clear to us. The model just assumes that the quantum brain is excited by the potential field set up by the classical brain. Another obvious question is that of decoherence. In this regard, we admit that the model proposed here is highly idealized since we have used Schroedinger wave equation. We intend to replace Schroedinger wave equation by density matrix approach in our future work. Also, the phase transition analysis of closed form dynamics, given in equation (6) with respect to various parameters $m, \zeta, \beta$ and $N$, has been kept for future work.

Finally, we believe that apart from the computational power derived from quantum computing, quantum learning systems will also provide a potent framework to study the subjective aspects of the nervous system [21]. The challenge to bridge the gap between physical and mental (or objective and subjective) notions of matter may be most successfully met within the framework of quantum learning systems. In this framework, we have proposed a notion of a quantum brain, and a Recurrent Quantum Neural Network has been hypothesized as a first step towards a neural computing model.
[1] M.A.Cohen and S. Grossberg, IEEE Trans Syst, Man and Cybernetics 13, 815 (1983).

[2] S. Amari, IEEE Trans SMC SMC-13, 741 (1983).

[3] L. Behera, M. Gopal, and S. Chaudhury, IEEE Trans Neural Networks 7, 1401 (1996).

[4] L. Behera, S. Chaudhury, and M. Gopal, IEE Proceedings Control Theory and Applications 145, 134 (1998).

[5] D. Amit, Modeling brain function (Springerverlag,Berlin/Heidelberg, 1989).

[6] J. A. Tuszynski, S. R. Hameroff, M. V. Sataric, B. Trpisova, and M. L. A. Nip, Journal of Theoretical Biology 174, 371 (1995).

[7] G. Vitiello, International Journal of Modern Physics B 9, 973 (1995).

[8] S. Hagan, S. R. Hameroff, and J. A. Tuszynski, http://arxiv.org/abs/quant-ph/0005025 (2000).

[9] A. Mershin, D. V. Nanopoulos, and E. Skoulakis, http://arxiv.org/abs/quant-ph/0007088 (2000).
[10] R. Penrose, Shadows of the Mind (Oxford University Press, 1994).

[11] L. Behera, B. Sundaram, G. Singhal, and M. Agarawal, IEEE Trans. Neural Networks (2003), revised and submitted.

[12] A. T. Bahill and L. Stark, Scientific American 240, 84 (1979).

[13] S. Gupta and R. Zia, Journal of Computer and System Sciences 63, 355 (2001).

[14] R. Boyd, Nonlinear Optics (Academic Press, 1991).

[15] E. A. Jackson, Perspectives of Nonlinear Dynamics (Cambridge, 1991).

[16] I. Bialynicki-Birula and J. Mycielski, Annals of Physics 100, 62 (1976).

[17] A. S. Davydov, Biology and Quantum Mechanics (Pergamon Press, Oxford, 1982).

[18] A. C. Scott, F. Y. F. Chu, and D. W. McLaughlin (1973), vol. 61 . 
[19] M. S. Grewal and A. P. Andrews, Kalman Filtering : Theory and Practice Using MATLAB (WileyInterscience, 2001).

[20] A. T. Bahill, M. J. Iandolo, and B. T. Troost, Vision
Research 20, 923 (1980).

[21] H. Atmanspacher, Discrete Dynamics 8, 51 (2004). 